\title{
How low can you go? Lowest effective dose of neuromuscular blocking agent for tracheal intubation
}

\author{
Aaron F. Kopman, MD
}

Published online: 2 May 2009

(C) Canadian Anesthesiologists' Society 2009

For most of the last half century, the holy grail of neuromuscular pharmacologists has been to develop a drug or a technique that can replace succinylcholine as the benchmark for rapid onset of neuromuscular block and that has, at the very least, the potential to promptly reverse its residual effects. With the neuromuscular blocking agent rapacuronium, it was established that ultra-fast onset and a nondepolarizing mechanism of action were not mutually incompatible. Unfortunately, rapacuronium was unacceptable because of serious side-effects. ${ }^{1}$ Rocuronium $1.2 \mathrm{mg} \cdot \mathrm{kg}^{-1}$, followed by sugammadex in appropriate dosage, can also be made to mimic the onset-offset profile of succinylcholine. ${ }^{2}$ Thus, the end of the "Era of Succinylcholine" is being announced at regular intervals, most recently by Lee, ${ }^{3}$ who this year opined, “...it finally appears that suxamethonium can be retired. Suxamethonium has done its job and has seen its day!" Perhaps; however, to paraphrase Mark Twain, "rumours of succinylcholine's demise appear to be greatly exaggerated."

Nevertheless, in this issue of the Journal, Siddik-Sayyid et $a .^{4}$ present an alternative to succinylcholine facilitated tracheal intubation, which some clinicians may find attractive and which, at its worst, provides the direction for additional clinical studies. Siddik-Sayyid et al. performed the following induction-intubation sequence. (1) After preoxygenation, the patients received lidocaine $1.5 \mathrm{mg} \cdot \mathrm{kg}^{-1}$ as a bolus, immediately followed by rocuronium $0.30 \mathrm{mg} \cdot \mathrm{kg}^{-1}$. (2) During the next $30 \mathrm{sec}$, the patients received remifentanil $2 \mu \mathrm{g} \cdot \mathrm{kg}^{-1}$ plus propofol

\footnotetext{
A. F. Kopman, MD ( $\square)$

Department of Anesthesiology, Weill Cornell College

of Medicine, 70 East 10th Street, Apt. 17F, New York, NY 10003, USA

e-mail: akopman@nyc.rr.com; akopman@gmail.com
}

$2.0 \mathrm{mg} \cdot \mathrm{kg}^{-1}$. (3) Laryngoscopy was initiated $60 \mathrm{sec}$ after completion of the anesthetic induction $(90 \mathrm{sec}$ after the rocuronium). The intubation scores were compared with a group that received succinylcholine $1.5 \mathrm{mg} \cdot \mathrm{kg}^{-1}$ immediately following an identical lidocaine-remifentanilpropofol sequence. Excellent intubating conditions were achieved in a similar proportion in both groups $(90 \% \mathrm{vs}$ $96 \%$, respectively).

Siddik-Sayyid et ll $^{4}$ are not the first to suggest that administering a non-depolarizing neuromuscular blocker before induction of anesthesia shortens the "intubating interval". The method has been called the "timing principle" ${ }^{5}$ Nor are they the first to tout the virtues of low-dose rocuronium (short duration of action). ${ }^{6,7}$ The strength of the authors' paper lies in its rigorous attention to detail. They used a randomized blinded prospective approach to their noteworthy study in comparing their proposed technique with the "gold standard" dose of succinylcholine $(1.5 \mathrm{mg}$. $\mathrm{kg}^{-1}$ ). In addition, the authors employed a well recognized method to evaluate conditions for intubation. Finally, when rocuronium was given as a single $\mathrm{ED}_{95}$ (effective dose for $95 \%$ block) bolus $\left(0.30-0.35 \mathrm{mg} \cdot \mathrm{kg}^{-1}\right), 90 \%$ of its peak effect at the adductor pollicis was reached in about 1.75 min. $^{8}$ Rocuronium's effect on those muscles that are more relevant to ease of intubation, such as the masseter and laryngeal adductors, likely occurred even sooner. Thus, the authors' timing sequence (total induction time $\approx 100 \mathrm{sec}$ ) was probably close to ideal.

The rationale for the authors' technique is based on the rather unique pharmacodynamic properties of rocuronium. At a single $\mathrm{ED}_{95}$ bolus, rocuronium is probably best thought of as a drug with a short rather than an intermediate duration of effect. In fact, in a recent study by Pendeville et al., ${ }^{9}$ the authors compared the duration of effect of rocuronium $0.30 \mathrm{mg} \cdot \mathrm{kg}^{-1}$ with that of mivacurium 
$0.15 \mathrm{mg} \cdot \mathrm{kg}^{-1}\left(2 \mathrm{ED}_{95}\right)$. There were 25 patients in each group. The results indicated that the two drugs had very similar recovery profiles. The clinical duration of rocuronium (time to $25 \%$ recovery of first twitch or T1) was $15 \pm 1 \mathrm{~min}$, and the time to spontaneous return of a trainof-four (TOF) ratio of 0.90 was $31 \pm 2 \mathrm{~min}$. Schlaich et $a .^{7}$ report a very similar clinical duration, although their recovery time to a TOF ratio of 0.80 was somewhat longer and with greater individual variability $(34 \pm 7 \mathrm{~min})$. Essentially identical values are reported by Prien et al. ${ }^{10}$ They report a clinical duration of $13.9 \pm 3.9$ min during propofol opioid anesthesia and $16.7 \pm 4.8$ min when anesthesia was supplemented with enflurane. Thus, while rocuronium $0.30 \mathrm{mg} \cdot \mathrm{kg}^{-1}$ has a longer duration of effect than succinylcholine $1.0-1.5 \mathrm{mg} \cdot \mathrm{kg}^{-1}$, satisfactory antagonism of rocuronium-induced residual block with neostigmine is clearly feasible within 15-20 min after rocuronium's administration, and reversal of its effects can probably be omitted if 45-60 min have passed from the time rocuronium was first administered. Thus, those clinicians who mourn the commercial withdrawal of mivacurium from the North American market would be well served to explore the versatility offered by low-dose rocuronium.

The authors' results are impressive, and I have no reason to doubt their validity; nevertheless, the paper has one serious weakness, i.e., the absence of a control group (no neuromuscular blocking agent). The authors contend that a control group was unnecessary since previous investigators have concluded that $2.0-4.0 \mu \mathrm{g} \cdot \mathrm{kg}^{-1}$ of remifentanil plus propofol in the absence of a neuromuscular blocker produces acceptable conditions for tracheal intubation in only about $50-60 \%$ of patients. ${ }^{11,12}$ However, other authors would disagree. Woods et al. achieved considerable success with remifentanil doses as low as $1 \mu \mathrm{g} \cdot \mathrm{kg}^{-1}$ when the drug was combined with lidocaine $1.5 \mathrm{mg} \cdot \mathrm{kg}^{-1} \cdot 13$ Thus, it remains unclear to what degree adding rocuronium contributed to the excellent intubation scores reported by the present authors.

The authors' data notwithstanding, it is probably premature to employ this protocol when a rapid sequence intubation is strongly indicated. In these clinical situations, succinylcholine $1.0-1.5 \mathrm{mg} \cdot \mathrm{kg}^{-1}$ or rocuronium 1.0 $1.2 \mathrm{mg} \cdot \mathrm{kg}^{-1}$ are still the established standards of care. If this technique is reserved for elective situations, then logical questions ensue. What's the rush? Is an inductionintubation interval of $60 \mathrm{sec}$ necessarily that critical? What's wrong with intervals of 75 or $90 \mathrm{sec}$ ? Therefore, while the authors' protocol was well planned and expertly administered, they omitted at least one intermediate step that should have preceded the current study.
No previous studies have examined intubating conditions following lidocaine $1.5 \mathrm{mg} \cdot \mathrm{kg}^{-1}$ plus remifentanil $2 \mu \mathrm{g} \cdot \mathrm{kg}^{-1}$ in the absence of a neuromuscular blocking drug. One can't help but wonder what the authors' results would have been if they had employed the present induction sequence in three different groups: Group 1: no neuromuscular blocking agent, laryngoscopy in $90 \mathrm{sec}$; Group 2: rocuronium $0.30 \mathrm{mg} \cdot \mathrm{kg}^{-1}$ immediately following the propofol, laryngoscopy in $90 \mathrm{sec}$; Group 3: succinylcholine $1.0-1.5 \mathrm{mg}$, laryngoscopy in 60 or $90 \mathrm{sec}$. I suspect that the more conventional sequence in Group 2 (rocuronium after induction) would be received more favourably by most clinicians, rather than administering rocuronium prior to loss of consciousness. If nothing else, it would have the virtue of being less prone to errors in technique and timing. Obviously, other sequences and protocols could also have been suggested. I recognize that the authors' objective was to achieve conditions for intubation within $60 \mathrm{sec}$ after induction of anesthesia and not to achieve conditions different from those produced by succinylcholine. I simply question whether this is an important goal in most clinical circumstances.

To what extent should we embrace the Siddik-Sayyid et $a l .{ }^{4}$ technique in our day-to-day practice? The intubation scores that they report are certainly impressive and comparable to those following succinylcholine. Nevertheless, a few additional caveats come to mind:

Propofol $2.0-2.5 \mathrm{mg} \cdot \mathrm{kg}^{-1}$ plus remifentanil $2 \mu \mathrm{g}$. $\mathrm{kg}^{-1}$ is not without potential cardiovascular repercussions. Mean blood pressure fell to $69 \pm 13 \mathrm{mmHg}$ after induction, and some patients required vasopressors. This finding indicates that some patients likely had mean pressures less than $50 \mathrm{mmHg}$ !

How practical is the technique? Propofol and remifentanil were administered simultaneously via two stopcocks over $30 \mathrm{sec}$. Does this technique require two hands or at least one infusion pump?

The authors' protocol is very specific as to its details. I suspect small variations in technique or timing may have significant consequences. Having watched countless residents and colleagues "personalize" the priming principle, I am wary of the potential for mishaps in applying this induction sequence. Someone somewhere will surely delay the rocuronium-propofol interval past $30 \mathrm{sec}$.

A final thought. It is clear that the term "intubating dose" has ceased to have meaning. The dose of neuromuscular blocking agent necessary to achieve good to excellent conditions for tracheal intubation is a function of the anesthetic induction technique, the onset profile of the drug, the timing of laryngoscopy, and the skill of the clinician. 


\section{Diminuer, diminuer... jusqu’à quel point? La plus petite dose efficace de curare pour l'intubation trachéale}

Depuis quelque cinquante ans, le Saint Graal des pharmacologues spécialisés en transmission neuromusculaire a été de mettre au point un médicament ou une technique qui pourrait remplacer la succinylcholine comme point de référence pour obtenir un délai d'installation rapide du bloc neuromusculaire, et qui aurait au moins le potentiel d'inverser rapidement ses effets résiduels. Le curare rapacuronium a permis d'établir qu'un délai d'installation ultrarapide n'était pas incompatible avec un mécanisme d'action non dépolarisant. Malheureusement, le rapacuronium s'est avéré un agent inacceptable en raison de ses effets secondaires graves. ${ }^{1}$ Le rocuronium $1,2 \mathrm{mg} \cdot \mathrm{kg}^{-1}$, suivi par du sugammadex à une dose adaptée, peut également reproduire le profil de délai d'installation/ récupération de la succinylcholine. ${ }^{2}$ Ainsi, la fin de « l'ère de la succinylcholine » est annoncée à intervalles réguliers, tout récemment par Lee, ${ }^{3}$ qui a déclaré cette année que « ... il semble finalement que nous pouvons retirer le suxaméthonium. Le suxaméthonium a accompli sa tâche et eu une belle vie ! » Peut-être en est-il ainsi ; toutefois, pour paraphraser Mark Twain, « les rumeurs concernant la mort de la succinylcholine semblent être très exagérées.»

Quoi qu'il en soit, dans ce numéro du Journal, SiddikSayyid et coll. ${ }^{4}$ présentent une alternative à la succinylcholine pour l'intubation trachéale, et certains cliniciens pourraient être séduits par cette technique; même si ce n'était pas le cas, cette étude ouvre la voie et donne une orientation possible à d'autres études cliniques. Siddik-Sayyid et coll. ont réalisé la séquence d'inductionintubation suivante : 1) Après la préoxygénation, les patients ont reçu un bolus de lidocaïne $1,5 \mathrm{mg} \cdot \mathrm{kg}^{-1}$, suivi immédiatement par du rocuronium $0,30 \mathrm{mg} \cdot \mathrm{kg}^{-1}$. 2) $\mathrm{Au}$ cours des $30 \mathrm{sec}$ suivantes, les patients ont reçu du rémifentanil $2 \mu \mathrm{g} \cdot \mathrm{kg}^{-1}$ plus du propofol $2,0 \mathrm{mg} \cdot \mathrm{kg}^{-1}$. 3) $\mathrm{La}$ laryngoscopie a commencé $60 \mathrm{sec}$ après avoir complété l'induction de l'anesthésie (90 sec après le rocuronium). Les scores d'intubation ont été comparés à ceux d'un groupe ayant reçu de la succinylcholine $1,5 \mathrm{mg} \cdot \mathrm{kg}^{-1}$ immédiatement après une séquence identique de lidocaïnerémifentanil-propofol. D'excellentes conditions d'intubation ont été obtenues dans une proportion comparable dans les deux groupes (90\% vs $96 \%$, respectivement).

Siddik-Sayyid et coll. ${ }^{4}$ ne sont pas les premiers à émettre l'hypothèse que l'administration d'un curare avant l'induction de l'anesthésie réduise « l'intervalle d'intuba- tion ». La méthode a été nommée le «principe de synchronisation ». ${ }^{5}$ Ils ne sont pas non plus les premiers à faire l'éloge des vertus du rocuronium à faible dose (durée d'action courte). ${ }^{6,7} \mathrm{La}$ force de l'article présenté ici réside dans son attention minutieuse aux détails. Les auteurs ont utilisé une approche prospective randomisée en aveugle dans leur étude remarquable, en comparant leur technique avec la dose «étalon or » de succinylcholine $\left(1,5 \mathrm{mg} \cdot \mathrm{kg}^{-1}\right)$. En outre, les auteurs ont fait appel à une méthode reconnue pour évaluer les conditions lors de l'intubation. Enfin, lorsque le rocuronium a été administré en bolus unique à une dose $\mathrm{ED}_{95}$ (dose efficace pour un bloc à $95 \%)\left(0,30\right.$ à $\left.0,35 \mathrm{mg} \cdot \mathrm{kg}^{-1}\right), 90 \%$ de son effet maximum à l'adducteur du pouce a été atteint dans un laps d'environ 1,75 min. $^{8}$ L'effet du rocuronium sur les muscles qui jouent un rôle important dans la facilité de l'intubation, tels que le masseter et les adducteurs du larynx, est possiblement survenu encore plus tôt. Ainsi, la séquence de synchronisation des auteurs (temps total d'induction $\approx 100 \mathrm{sec}$ ) était probablement proche de la séquence idéale.

La justification de la technique des auteurs repose sur les propriétés pharmacodynamiques quelque peu spéciales du rocuronium. Lorsqu'il est administré en bolus unique à une dose de $\mathrm{ED}_{95}$, le rocuronium peut probablement être considéré comme un agent à la durée d'action courte plutôt qu'intermédiaire. En fait, dans une étude récente réalisée par Pendeville et coll., ${ }^{9}$ les auteurs ont comparé la durée d'action du rocuronium $0,30 \mathrm{mg} \cdot \mathrm{kg}^{-1}$ à celle du mivacurium $0,15 \mathrm{mg} \cdot \mathrm{kg}^{-1}\left(2 \cdot \mathrm{ED}_{95}\right)$. L'étude de Pendeville comptait 25 patients dans chaque groupe. Les résultats ont indiqué que les deux agents présentaient des profils de récupération similaires. La durée clinique du rocuronium (temps jusqu'à une récupération de $25 \%$ du premier élément du train-de-quatre, ou T1) était de $15 \pm 1 \mathrm{~min}$, et le temps nécessaire à la récupération spontanée du rapport T4/T1 à 0,90 était de $31 \pm 2$ min. Schlaich et coll. ${ }^{7}$ rapportent une durée clinique équivalente, bien que dans leur étude le temps de récupération jusqu'à un T4/T1 de 0,80 était un peu plus long et présentait une variabilité plus prononcée entre les individus (34 $\pm 7 \mathrm{~min}$ ). Prien et coll. ${ }^{10}$ rapportent fondamentalement les mêmes valeurs. Ces auteurs font état d'une durée clinique de 13,9 \pm 3,9 min pendant une anesthésie réalisée avec un opioïde et du propofol et de 16,7 \pm 4,8 min lorsque de l'enflurane a été ajouté à l'anesthésie. Par conséquent, bien que le rocuronium $0,30 \mathrm{mg} \cdot \mathrm{kg}^{-1}$ ait une durée d'action prolongée par rapport à la succinylcholine 1,0 à $1,5 \mathrm{mg} \cdot \mathrm{kg}^{-1}$, un antagonisme satisfaisant du bloc résiduel provoqué par le rocuronium peut clairement être réalisé avec de la néostigmine dans les 15 à 20 min après l'administration du rocuronium, et l'inversion de ses effets peut ne pas être 
nécessaire si 45 à 60 min se sont écoulées depuis l'administration initiale du rocuronium. Dès lors, les cliniciens qui déplorent le retrait commercial du mivacurium des marchés nord-américains pourraient être séduits par la polyvalence que le rocuronium à faible dose offre.

Les résultats des auteurs de l'étude présentée ici sont pour le moins impressionnants, et je n'ai aucune raison de remettre en question leur validité ; toutefois, l'article a une faiblesse majeure, soit l'absence d'un groupe témoin (pas de curare). Selon les auteurs, un groupe témoin était inutile étant donné que d'autres chercheurs avaient déjà par le passé conclu que du rémifentanil 2,0 à $4,0 \mu \mathrm{g} \cdot \mathrm{kg}^{-1}$ combiné à du propofol en l'absence d'un curare créait des conditions adéquates pour l'intubation trachéale chez seulement 50 à $60 \%$ des patients. ${ }^{11,12}$ Cependant, il faut noter que d'autres auteurs contestent ces données. Woods et coll. ont rencontré un succès remarquable avec des doses de rémifentanil aussi basses que $1 \mu \mathrm{g} \cdot \mathrm{kg}^{-1}$ lorsque l'agent était combiné à de la lidocaïne $1,5 \mathrm{mg} \cdot \mathrm{kg}^{-1} \cdot{ }^{13}$ Dès lors, nous ne pouvons déterminer clairement dans quelle mesure le fait d'ajouter du rocuronium a réellement contribué aux scores d'intubation excellents rapportés par les auteurs de l'étude présentée ici.

En dépit des données intéressantes présentées par SiddikSayyid et coll., ${ }^{4}$ il est probablement trop tôt pour employer ce protocole lorsqu'une intubation en séquence rapide est vivement recommandée. Dans de telles situations cliniques, la succinylcholine 1,0 à $1,5 \mathrm{mg} \cdot \mathrm{kg}^{-1}$ ou le rocuronium 1,0 à $1,2 \mathrm{mg} \cdot \mathrm{kg}^{-1}$ demeurent les normes de soins établies. Mais si cette technique devait être réservée aux situations non urgentes, plusieurs questions logiques se posent. Pourquoi tant de hâte ? Un intervalle d'induction-intubation de $60 \mathrm{sec}$ est-il vraiment crucial à ce point ? Quel est le problème avec des intervalles de 75 ou $90 \mathrm{sec}$ ? Par conséquent, quoique le protocole des auteurs était bien planifié et administré de façon experte, les auteurs ont omis au moins une étape intermédiaire qui aurait dû précéder cette étude.

À ma connaissance, aucune étude antérieure n'a examiné les conditions d'intubation après l'administration de lidocaïne $1,5 \mathrm{mg} \cdot \mathrm{kg}^{-1}$ plus rémifentanil $2 \mu \mathrm{g} \cdot \mathrm{kg}^{-1}$ en l'absence de curare. Dès lors, la question qui nous titille est : quels résultats les auteurs auraient-ils obtenu s'ils avaient utilisé cette séquence d'induction dans trois groupes différents ? Soit groupe 1 : pas de curare, laryngoscopie en $90 \mathrm{sec}$; groupe 2 : rocuronium $0,30 \mathrm{mg} \cdot \mathrm{kg}^{-1}$ immédiatement après le propofol, laryngoscopie en $90 \mathrm{sec}$; et groupe 3 : succinylcholine 1,0 à $1,5 \mathrm{mg}$, laryngoscopie en $60 \mathrm{ou} 90 \mathrm{sec}$. Selon moi, la séquence plus traditionnelle administrée au groupe 2 (rocuronium après l'induction) recevrait un accueil favorable de la plupart des cliniciens, à la place d'administrer le rocuronium avant la perte de conscience. Quoi qu'il en soit, cette méthode serait moins susceptible aux erreurs de technique ou de synchronisation. Bien évidemment, nous aurions pu proposer d'autres séquences et protocoles. Je suis conscient que l'objectif des auteurs était de créer des conditions satisfaisantes pour l'intubation dans un délai de 60 sec après l'induction de l'anesthésie, non pas de créer des conditions différant de celles offertes par la succinylcholine. Ce que je remets en question, c'est la pertinence de cet objectif pour la plupart des circonstances cliniques.

Dans quelle mesure devrions-nous adopter la technique proposée par Siddik-Sayyid et coll. ${ }^{4}$ dans notre pratique quotidienne ? Les scores d'intubation qu'ils rapportent sont pour le moins impressionnants et comparables à ceux réalisés avec la succinylcholine. Néanmoins, d'autres éléments devraient aussi être pris en considération par le clinicien avant d'adopter une telle technique :

Le propofol, de 2,0 à $2,5 \mathrm{mg} \cdot \mathrm{kg}^{-1}$, plus rémifentanil $2 \mu \mathrm{g} \cdot \mathrm{kg}^{-1}$ n'est pas sans répercussions cardiovasculaires potentielles. La pression artérielle moyenne a chuté à $69 \pm 13 \mathrm{mmHg}$ après l'induction, et il a fallu administrer des vasopresseurs à certains patients. Ces données indiquent que certains patients avaient probablement des pressions moyennes de moins de $50 \mathrm{mmHg}$ !

Cette technique est-elle pratique ? Le propofol et le rémifentanil ont été administrés simultanément via deux robinets à trois voies pendant un laps de $30 \mathrm{sec}$. Cette technique nécessite-t-elle les deux mains ou au moins une pompe à perfusion?

Le protocole de Siddik-Sayyid et coll. est très spécifique quant à ses détails. Je suppose que de légères variations dans la technique ou dans la synchronisation des différents gestes pourraient avoir des conséquences majeures. Ayant vu d'innombrables résidents et collègues «personnaliser » le principe d'amorçage, je crains que cette séquence d'induction comporte un important potentiel d'incidents dans son application. Quelqu'un, quelque part, va certainement prolonger l'intervalle de rocuronium-propofol audelà de $30 \mathrm{sec}$.

En conclusion, il est clair que le terme de " dose d'intubation » a perdu toute signification. La dose de curare nécessaire pour créer des conditions d'intubation trachéale bonnes à excellentes est fonction de la technique d'induction de l'anesthésie, du profil d'installation de l'agent, de la synchronisation de la laryngoscopie, et de la compétence du clinicien.

Conflicts of interest During the past year I have served as an ad hoc consultant to Schering-Plough/Organon Pharmaceuticals. I have also served as a member of their speakers' bureau.

\section{References}

1. Wierda JM, van den Broek L, Proost JH, Verbaan BW, Hennis PJ. Time course of action of endotracheal intubating conditions of Org 9487, a new short-acting steroidal muscle relaxant; a comparison with succinylcholine. Anesth Analg 1993; 77: 579-84. 
2. Puhringer FK, Rex C, Sielenkamper AW, et al. Reversal of profound, high-dose rocuronium-induced neuromuscular blockade by sugammadex at two different time points: an international, multicenter, randomized, dose-finding, safety assessor-blinded, phase II trial. Anesthesiology 2008; 109: 188-97.

3. Lee C. Goodbye suxamethonium! Anaesthesia 2009; 64 Suppl 1: 73-81.

4. Siddik-Sayyid SM, Taha SK, Kanazi GE, et al. Excellent intubating conditions with low-dose rocuronium or succinylcholine, remifentanil-propofol. Can J Anesth 2009; 56.

5. Cicala $R$, Westbrook $L$. An alternative method of paralysis for rapid sequence induction. Anesthesiology 1988; 69: 983-6.

6. Barclay K, Eggers K, Asai T. Low-dose rocuronium improves conditions for tracheal intubation after induction of anaesthesia with propofol and alfentanil. Br J Anaesth 1997; 78: 92-4.

7. Schlaich N, Mertzlufft F, Soltesz S, Fuchs-Buder T. Remifentanil and propofol without muscle relaxants or with different doses of rocuronium for tracheal intubation in outpatient anaesthesia. Acta Anaesthesiol Scand 2000; 44: 720-6.

8. Kopman AF, Klewicka MM, Kopman DJ, Neuman GG. Molar potency is predictive of the speed of onset of neuromuscular block for agents of intermediate, short, and ultrashort duration. Anesthesiology 1999; 90: 425-31.

9. Pendeville PE, Lois F, Scholtes JL. A comparison of intubation conditions and time-course of action with rocuronium and mivacurium for day case anaesthesia. Eur J Anaesthesiol 2007; 24: 546-50.

10. Prien T, Zahn P, Menges M, Brussel T. 1x ED90 dose of rocuronium bromide: tracheal intubation conditions and time-course of action. Eur J Anaesthesiol Suppl 1995; 11: 85-90.

11. Stevens $J B$, Wheatley $L$. Tracheal intubation in ambulatory surgery patients: using remifentanil and propofol without muscle relaxants. Anesth Analg 1998; 86: 45-9.

12. Klemola UM, Mennander S, Saarnivaara L. Tracheal intubation without the use of muscle relaxants: remifentanil or alfentanil in combination with propofol. Acta Anaesthesiol Scand 2000; 44: 465-9.

13. Woods AW, Grant S, Harten J, Noble JS, Davidson JA. Tracheal intubating conditions after induction with propofol, remifentanil and lignocaine. Eur J Anaesthesiol 1998; 15: 714-8. 